\title{
Studies on Single Neurons in Dorsal Hippocampal Formation and Septum in Unrestrained Rats
}

\author{
Part II. Hippocampal Slow Waves and Theta Cell Firing \\ During Bar Pressing and Other Behaviors
}

\author{
Robert Feder and James B. Ranck, Jr.
}

The University of Michigan Medical School, Medical Science Building, Ann Arbor. Michigan 48104

Receized May 9, 1973

\section{INTRODUCTION}

The past decade has seen a great concentration of work on behavioral correlates of hippocampal slow-wave theta rhythm. A number of theories have been advanced to explain the behavioral significance of theta, with that of Vanderwolf bcing the least vague and most extensively studied in the rat. His theory also comes closest to describing the data behavioristically, rather than implying internal mechanisms, so it will be the easiest to use as a frame of reference, especially in the rat. Vanderwolf (31) has proposed that hippocampal slow-wave theta is a concomitant of voluntary movement in the rat. He found theta during walking, rearing, postural adjustments, approach to food and water, struggling when handled, head movements (orienting), and climbing. Desynchronized, irregular hippocampal slow waves were found during most motionlessness and "automatic" or consummatory behaviors, such as eating, drinking, teeth chattering, and grooming. If an automatic and a voluntary behavior occurred simultancously, theta was found in the slow waves. Vanderwolf defined voluntary behaviors as variable behavior sequences which can be conditioned by using a wide variety of operant reinforcers. Automatic behaviors were defined as instinctual fixed patterns of behavior limited to the motivational system in which they normally occur (31). His results have been substantiated by a number of investigators, all working with the rat $(10,15,19-22,24,25$, 27, 29). Sainsbury (28), using guinea pigs, confirmed Vanderwolf's 1969 results (30), and in addition found that urination, defecation, pelvic thrusting, ejaculation, and squealing all gave irregular hippocampal slow waves, while flight and pursuit in feuding males gave theta. Irmis, Madlafousek, and Hlinak (14) found consummatory sexual behaviors in rats elicited 
irregular hippocampal slow waves. Wishaw and Vanderwolf (32) found that shivering in the rat was accompanied by irregular hippocampal slow waves, while swimming was accompanied by theta. Yoshii, Shimokochi, Miyamoto, and Ito (35) found hippocampal slow waves in $\operatorname{logs}$ to be irregular during eating, defecation, urination, motionlessness, and barking, while theta was present during hearl movements (orienting), walking. and exploring. Dalton and Black (8), Dalton ( $\tau$ ), and Black, Young, and Batenchuk (6) showed that curarized dogs could be trained to give or refrain from giving theta in their hippocampal slow waves, this eliminating proprioceptive feedback as a source of theta activity.

The Vanderwolf hypothesis concerning the mechanism of hippocampal theta raises the question of whether or not a clearly voluntary, gross motor pattern will be accompanied by hippocampal theta once the motor pattern becomes stereotyped and "automatic" through intensive operant training. According to Wishaw and Vanderwolf (3.3), if the behavior to be conditioned is anything but motionless or a natural automatic (according to Vanderwolf's definition) behavior, it should be accompanied by theta regardless of how well learned it is. The literature which exists on the point is conflicting. Grastyan, Lissak, Madrasy, and Donhoffer (12) found that when cats were trained to go to a food chute to obtain food after the presentation of a CS, hippocampal theta was prominent in the early stages of learning but disappeared completely with training. The laboratory of Adey has produced a number of studies $(1-4,9)$ in which cats were trained to run down an alley and choose one of two doors, which was randomly illuminated, for a food reward. In this task, the presence of hippocampal theta persisted lhroughout ligh degrees of training. Vanderwolf (30) trained rats to escape from shock by jumping out of a box. After 300 trials over 10 days, when the response had become very stereotyped, theta was still elicited in the hippocampal slow waves during jumping. Yoshii et al. (34) found irregular hippocampal activity in dogs when an animal put a foreleg in the experimenter's hand upon verbal instruction. Lopes da Silva and Kamp (18) conditioned two dogs to press a bar for food reward after the presentation of a discrininative stimulus. In this study, hippocampal slow-wave theta was found during the dog's approach to the bar and the dog's walking away from the food chute after reinforcement, but not during bar-pressing itself. These results persisted for 2500 trials over 6 mo. Dalton and Black (8) found that dogs conditioned to press a bar at least once a second for $4-8 \mathrm{sec}$ to avoid shock exhibited hippocampal theta slow waves throughout training during the actual bar-press response. Training in this situation lasted for roughly 60 trials over 3 days. Wishaw and Vanderwolf (33) found hippocampal theta during the actual bar-press response on con- 
tinuous food reinforcement in rats over a period of a few days. At certain electrode sites (CA4 and fascia dentata), however, theta was seen in the slow waves only on the first day of training of the bar-press response. These authors also found that rats forced to run in a motor-drive wheel continued to show theta in the hippocampal slow waves after 10 days of $8 \mathrm{hr}$ of nonstop running per day. Pond and Schwartzbaum (23) found predominantly irregular hippocampal slow waves with occasional occurrences of theta, in rats pressing on a fixed-ratio 16 schedule for sucrose solution after 10 days of 20-min trials per day. All of the trained behaviors employed in the above studies would be classified as voluntary behaviors by Vanderwolf (31).

Vanderwolf (30) and Wishaw and Vanderwolf (33) have pointed out that the discrepancies in the above results may have been due to recording electrode placement, in that certain placements within the hippocampus of the rat gave no theta at all, while others gave theta only under very intense, vigorous activity involving a large muscular field. There is also no real agreement across studies as to exactly what constitutes theta. Some experimenters consider a peak in the theta range of the power spectrum of the slow waves as evidence of theta, others rely on simple visual inspection of polygraph records, others use computer averaging. It is conceivable that these problems could be avoided by recording directly from cells involved in the theta mechanism rather than recording the epiphenomenal slow waves themselves.

Ranck (24) has shown that a hippocampal neuron type, the "theta cells," fire at their most rapid rate if and only if there is a slow-wave theta rhythm. Most of these theta cells fire in rhythmical bursts phasc-locked to the hippocampal slow waves during all and only those behaviors which give theta in the slow waves. Ito and Olds (17) studied neurons during self-stimulation from medial forebrain bundle sites. The cells they recorded from in hippocampus had firing rates of from 4 to $64 / \mathrm{sec}$ averaging $24 / \mathrm{sec}$ during "locomotor activity," and thus most of them were theta cells. Indeed, they comment that many fired in groups that were at the theta frequency (they did not measure slow waves). During self-stimulation the rate of most hippocampal neurons slowed or stopped, and some rhythmical firing was present in the intervals between bar presses (behavior not described). Ito (16) had previously shown that self-stimulation was usually accompanied by $30-40 / \mathrm{sec}$ waves in the hippocampus, and that a regular theta rhythm was often present in the interval between bar presses. Lacking knowledge of interbar press behavior, it is difficult to compare those results to the rsults in the present study.

The present study was a direct attempt to determine the activity of the hippocampal theta mechanism during a well-learned, stereotyped voluntary 
motor response by recording both slow waves and action potentials from theta cells. The investigation of unit responses involved refinement of the description of firing characteristics of theta units begun by Ranck (2t) and the application of this information to a quantified analysis of the theta morle. Hippocampal slow-wave data were taken through the same movable microelectrode which was used to record units, thus slow waves were obtained from a variety of electrode loci in each animal. Par-pressing on both continuous reinforcement and fixed-ratio 50 serverl as well-learned, stereotyped, voluntary motor responses.

\section{METHODS}

Most of the methods have heen described (24). Five $300-450 \mathrm{~g}$ male Sprague-Dawley albino rats, 2-3 mo old at the start of the study, were used. Animals were caged with their littermates until surgery for electrode implantation. After surgery, animals were housed individually in glass aquaria in an open laboratory setting.

Training Schedule and Conditions. Animals were allowed 7-10 clays to recover from surgery and to display normal eating and drinking habits. They were then started on a bar-press training schedule. Animals 111 and 122 were trained on a water reward. Animals 110,121, and 123 were trained on a food reward. Animals started out on continuous reinforcement (CRF) and gradually worked up to fixed-ratio 50 (FR-50) within 7 clays. After 2-5 days of FR-50 performance, the animal was put on a mixed FR-50$\mathrm{CRF}$ schedule, with a glass jar inserted into the running box during CRF periods. The method of running was usually FR-50-CRF-FR-50-CRF, with each of the four periods lasting roughly 75 reinforcements for food reward, and 30 reinforcements for water reward. All four periods together lasted 60-90 min, by which time bar-pressing rates clecreased considerably and animals had had enough reinforcement to maintain body weight. Animals were always run approximately $22 \mathrm{hr}$, food or water deprived, since they received all of their food or water during bar pressing or immediately afterward. This training phase proceeded for at least $5 \mathrm{wk}$, after which time recording was begun. The food bar was located along the back wall of the running box; the water bar, along a side wall. The reinforcement deliveries (a clute for food and a dipper for water) were locaterl $8 \mathrm{~cm}$ to the left of their respective bars. During all training periods a recording of noises similar to those which are produced during unit recording was played over a loudspeaker to habituate the animal to these sounds. Also, a nonfunctional head stage was attached to the animal cluring training to habituate the rat to the head stage necessary cluring recording. The "openness" of the animal to the external environment harl 10 observable deleterious effect on its bar-pressing behavior. All animals produced cumulative records of classic appearance. 
Recording Procedure. The recording equipment was identical to that previously described (24), with the following alterations. An event pulse indicating a bar-press was electronically mixed onto the unit recording. Another event pulse indicating a reinforcement was electronically mixed onto the hippocampal slow-wave recording. All hippocampal slow-wave and unit recording was placed on magnetic tape for later play-back and analysis. A Grass polygraph was used for simultaneous recording of cortical EEG, hippocampal slow waves, and bar-press and reinforcement markers.

$\Lambda$ fter the 5 -wk training period, when bar pressing had become very stereotyped and well learned, unit recordings were taken in the running box. At the start of the recording session, the bar and delivery which the rat was trained on were covered. The microelectrode was continuously lowered into the brain until a theta cell was recognized and well isolated. ${ }^{1}$ In the course of locating theta cells, slow-wave records could be taken from a variety of loci. When a theta cell was well isolated, it was observed for at least 5 min to make sure a good isolation was obtained and that the cell was not injured. Data were then taken from the cell and hippocampal slow waves with the animal performing various behaviors, including lying motionless, approaching food or water, eating, drinking, grooming, walking, orienting, following the experimenter's hand around the inside of the box, orienting to a moving pencil, and being picked up in the experimenter's hand. These behaviors served as baseline measures for the hippocampal slow waves and the cell on well-known theta and non-theta behaviors. The cover over the bar and delivery was then removed, and the animal went through its bar-press routine. Data were taken at various times throughout approximately $1 \mathrm{hr}$ of bar-press behavior.

If a theta cell could not be isolated within $3 \mathrm{hr}$ from the start of a day's run, the animal was allowed access to the bar and only slow-wave data were taken for that day.

A single microelectrode was used until its track went entirely through the dorsal hippocampus. ${ }^{2}$ The frequency of failures of isolate theta cells usually necessitated two or three tracks on each side of an animal. Each animal was run for approximately 12 recording sessions.

Mcthods of Analysis. Unit and hippocampal slow wave recordings were analyzed by simple visual inspection. Unit recordings were also passed through a window discriminator and changed to standard spikes (discriminator output) with no background noise. From these filtered records, spike integration records were obtained, and frequency of firing was evaluated with an electronic counter. Values for frequency of firing on any particular behavior (except bar pressing) were obtained from approximately

\footnotetext{
${ }_{1}$ Since theta neurons constitute a minority of the total neuronal population of the hippocampus, many nontheta hippocampal cells were encountered, but not studied.

${ }^{2}$ A single track could yield several theta cells over a number of days.
} 
$45 \mathrm{sec}$ of data on that particular behavior. Values for bar pressing were obtained from much longer runs of data (up to $30 \mathrm{~min}$ ) taken throughout the bar-pressing session.

\section{RESULTS}

Sloz'-Waz' $e$ Recordings. Because of the small electrode tip used to record both mits and gross hippocampal slow waves, good slow-wave tracings were sometimes difficult to obtain, due to great amounts of movement and other artifacts. Thus, hippocampal slow-wave recordings were sometimes not obtained on days on which a unit record was successfully taken. However, satisfactory hippocampal slow-wave recordings were eventually obtained from all animals. Each animal yielded good slow-wave data during general behaviors from an average of 15 different electrode placements on 5 different days. Slow-wave data during bar-pressing was obtained from one placement per day for an average of 5 days per animal.

Electrode placement was a critical factor in how much theta activity could be recorded. Placements in CA4 and placements in stratum radiatum and stratum lacunosum-moleculare of CA1 and CA3 gave 1-2 mv slow waves witl good theta in certain behavioral situations (Figs. 1-3), whereas placements in stratum oriens and stratum pryamidale of fields CA1 and CA3 gave $200-600 \mu \mathrm{v}$ slow waves with little or no theta in any behavioral situations (Fig. 4). Dorsal fascia dentata placements gave 1-3 mv slow waves with some placements giving good theta, others giving little or no theta. The general behavioral concomitants of hippocampal slow-wave theta in those electrode sites which gave good theta are the same as the results of Vanderwolf (30). Motionlessness and the consummatory behaviors of eating, drinking, teeth chattering, vomiting, yawning, and grooming yielded irregular hippocampal slow waves, whereas voluntary behaviors like walking, orienting (head movements), approaching food or water, following the experimenter's hand, jumping, struggling while being held in the experimenter's hand, and exploring a novel object yielded clear slow wave theta rhythm (Figs. 1-3).

The form of the behavior on continuous reinforcement bar pressing was very similar for all animals on food reinforcement. The rat would typically keep one paw on or near the bar at all times. After a press, the animal quickly extended the upper part of its body over to the food clute, picked up the pellet, moved its head back toward the bar, and consumed the reward. The cycle was then repeated. Continuous reinforcement pressing for animals on water reward was similar, except that the animal had to move its whole body over to the dipper to drink the reward, then walk back to the bar for another press. Fixed-ratio 50 behavior took the following form for all animals: The rat would keep one paw on the bar and shake the entire npper portion of its trunk very vigorously. After 50 presses and the noise of the 

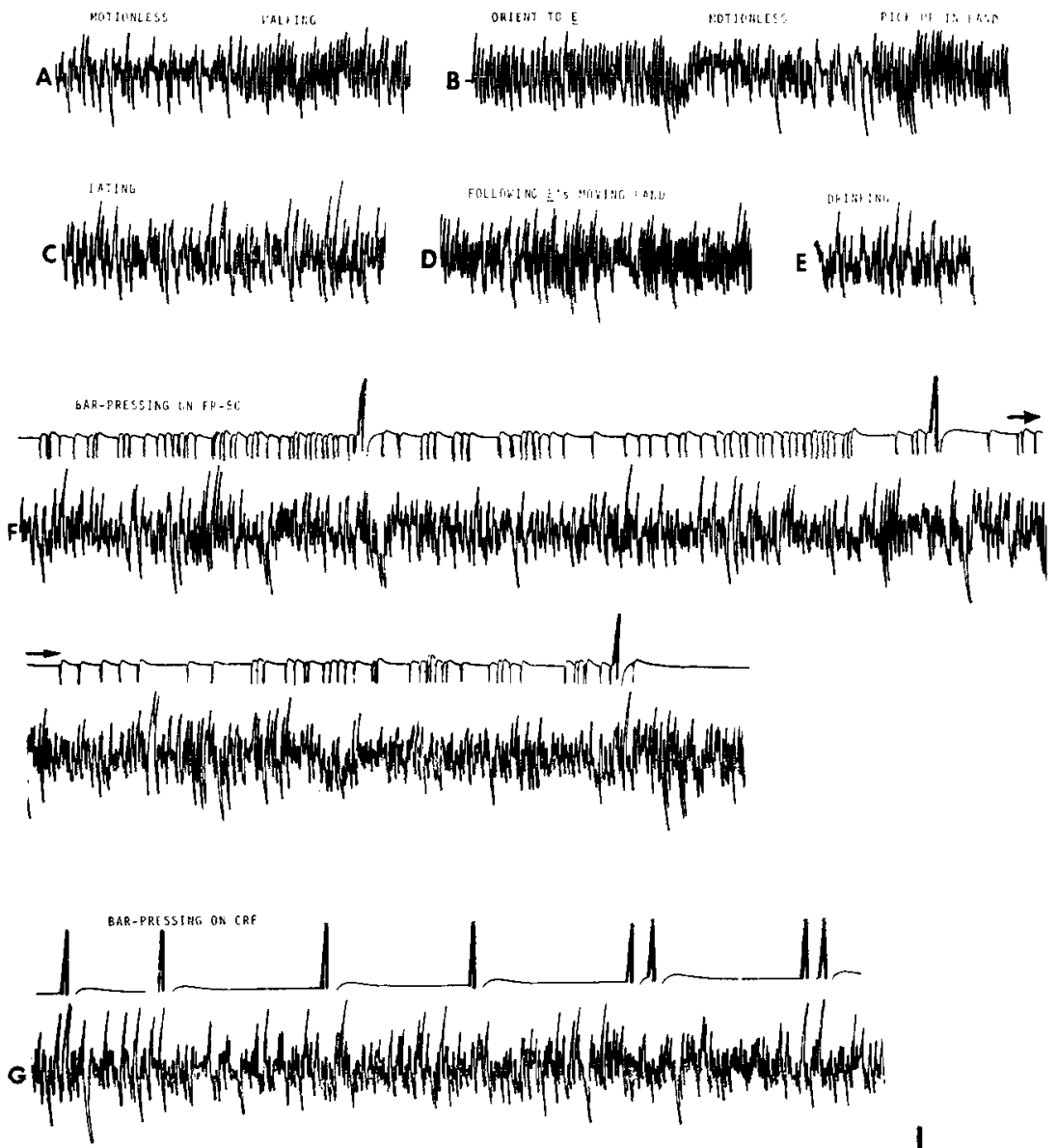

Fig. 1. Slow waves from animal 110 from an electrode placement in CA4. Clear theta is seen during walking, orienting (head movement), being picked up in the experimenter's hand, and following the experimenter's hand around the running box. Irrcgular activity is seen during motionlessness, eating, and drinking. In this and all subsequent figures, each lettered run of data is continuous. An arrow at the end of one line and another arrow at the beginning of the next indicate that the data is continuous. In $F$ and $G$, the tracing running above the slow waves indicates bar presses (small downward deflections) and reinforcements (large upward deflections). Note irregular slow wave activity during bar pressing on both continuous reinforcement (CRF) and fixed-ratio 50 (FR-50). Calibration marks: $5 \mathrm{sec} ; 1 \mathrm{mv}$ in Figs. 1-5.

magazine, the animal would quickly walk over to the reward, consume it, slowly come back into the immediate vicinity of the bar, and start pressing again. Postural changes during actual bar pressing occurred occasionally.

Bar-pressing on both continuous reinforcement and fixed-ratio 50 yielded predominantly irregular hippocampal slow wave records in all animals 

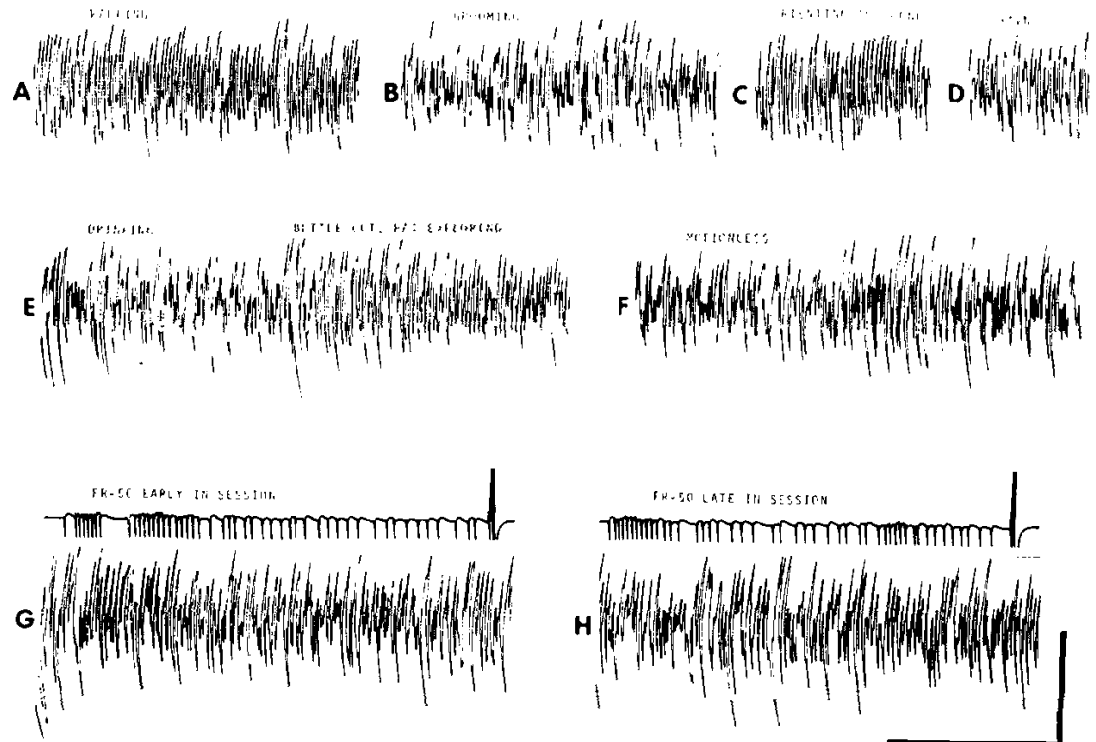

FIG. 2. Slow waves from animal 121 from a placement in stratum lacunosummoleculare of CA1. Walking, orienting, and exploring are accompanied by theta, while grooming, yawning, drinking, and motionlessness are accompanied by irregular activity. Bar pressing on FR-50 gives irregular slow waves both early and late in the bar-pressing session.

(Figs. 1-3). Occasionally, very brief $(1-3 \mathrm{sec})$ periods of theta could be found in the slow-wave records during fixed-ratio 50 bar pressing. These were usually associated with postural adjustments. The predominantly irregular slow waves were present throughout bar pressing from early (23 hr deprivation) to late (approaching satiation) in the bar-pressing session.

With an electrode placement 1 day in stratum radiatum of CA1, animal 122 showed regular theta in the slow waves for approximately the first 15 presses in every fixed-ratio 50 sequence, then gave a completely irregular record for the remaining 35 presses until reinforcement. However, on other days, with electrode placements giving good theta in CA4 and stratum radiatum and stratum lacunosum-moleculare of $\mathrm{CA} 3$, the slow waves were irregular throughout the entire phase of bar pressing. Slow-wave records during extinction were obtained from this same animal with an electrode placement in stratum lacunosuns-moleculare of CA3. Again, irregular slow waves were found to predominate during bar pressing on extinction (Fig. 3).

There was one anomalous finding with respect to slow waves. Animal 110 showed clearly irregular slow waves during bar pressing on various days from placements yielding good theta in fascia dentata, CA1, CA3, and 
A
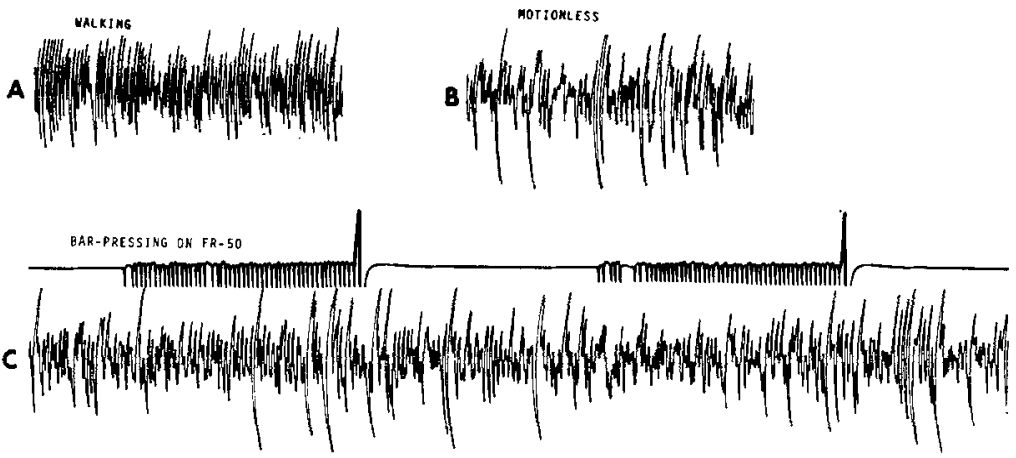

EXIINCTION - EART Y
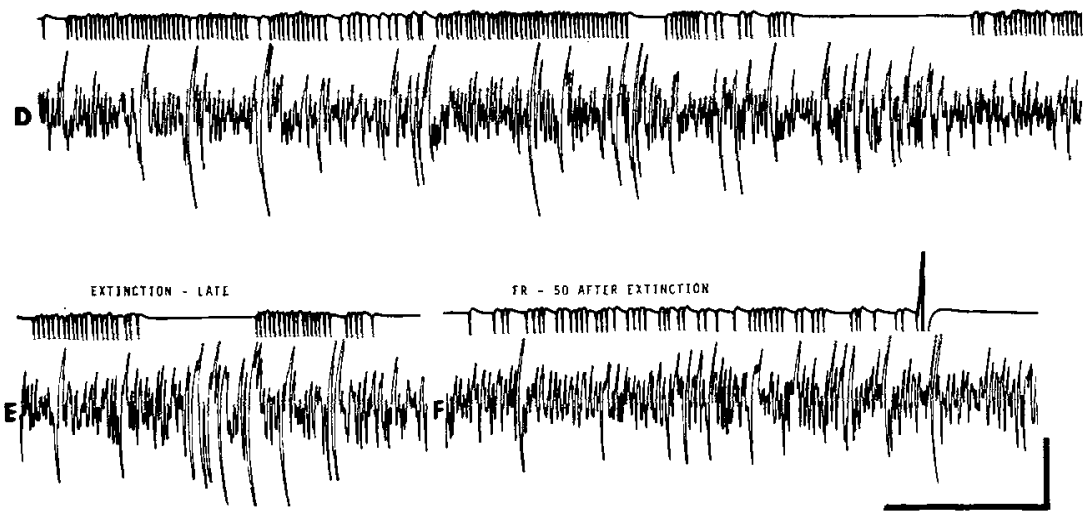

FIG. 3. Slow waves from animal 122 from an electrode placement in stratum lacunosum-moleculare of CA3. Bar pressing on FR-50 (run C) gives predominantly irregular slow waves. Run D shows predominantly irregular slow waves during extinction pressing. This run was taken early in the extinction period, $90 \mathrm{sec}$ and 400 bar presses after reinforcement had been withdrawn. Run $E$ shows continued irregular slow wave activity later in the extinction period, $20 \mathrm{~min}$ and 1400 bar presses after reinforcement had been withdrawn. Soon after the data in run $E$ was taken, reinforcement was reinstated on an FR-50 basis. Run F shows irregular slow wave activity during bar pressing on the second reinforcement of this reinstated FR-50.
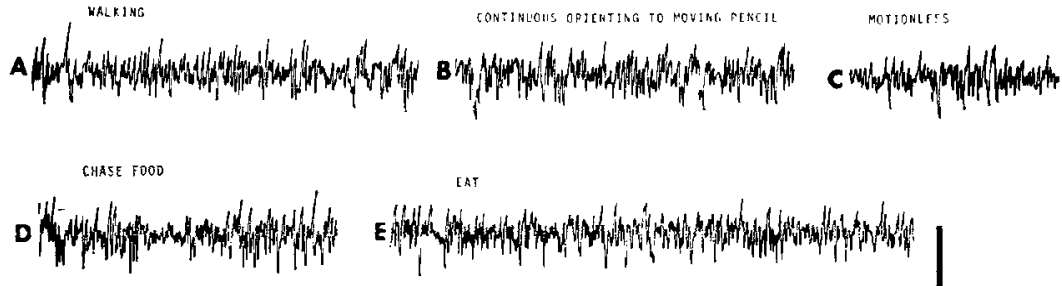

FIG. 4. An exceptional case. Slow waves during various behaviors in animal 122 from an electrode placement in stratum pyramidale of CA.3. This placement gave very little theta activity in the slow waves at any time, including during voluntary behaviors (walking, orienting, and chasing food). 

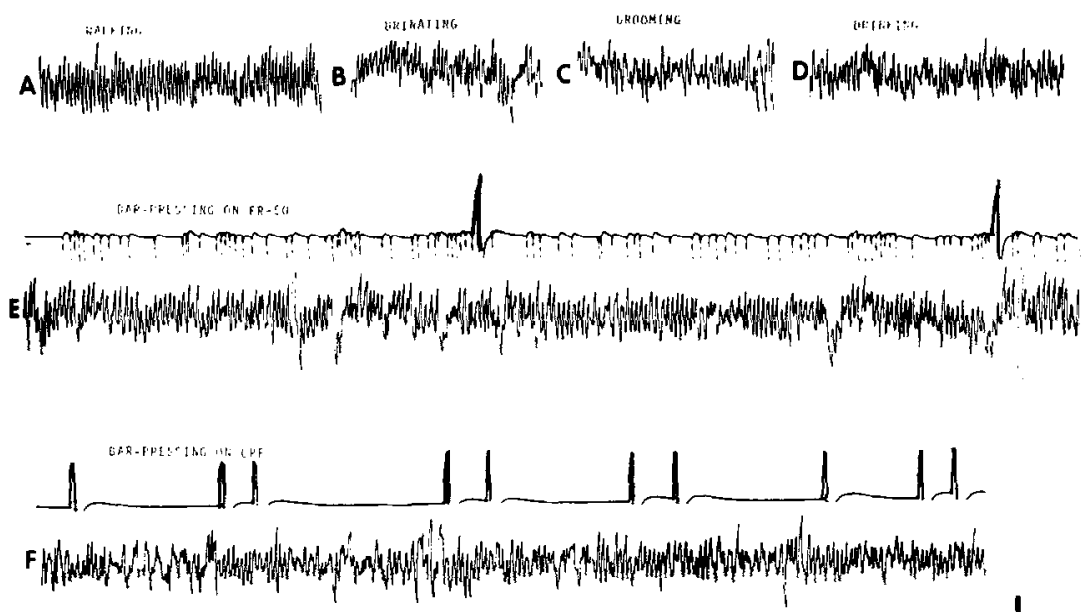

FIG. 5. An exceptional case. Slow waves from animal 110 from an electrode placement in CA4. Good theta is seen during walking, and bar pressing on CRF and FR-50. But also note the anomalous finding of theta during the consunmatory behaviors of urinating, grooming, and drinking.

CA4. However, on one day's run, an electrode placement in CA4 yielded clear theta during drinking, eating, urinating, grooming, and bar pressing on both continuous reinforcement and fixed-ratio 50 (Fig. 5). The animal's observable behavior on this day was no different from any of the other days on which theta was not seen in the slow waves during bar pressing and the consummatory behaviors.

Unit Recordings. Recorlings from 16 well-isolated theta-type units were obtained. Subject numbers 111, 121, and 123 yielded one unit each, and subject numbers 110 and 122 yielded 8 and 5 units, respectively. Histology revealed all units to be located in the dorsal hippocampal sections of CA1, 5 units, CA3, 4 units, CA4, 2 units, and fascia dentata, 5 units. Units were located primarily in stratum pryamidale of Anmon's horn and stratum granulosum of fascia dentata, but some units were found in stratum oriens, stratum radiatum, and stratum moleculare of fascia dentata.

Units usually could be held for over $1 \mathrm{hr}$. All units had spontaneous rates greater than $10 \mathrm{spike} / \mathrm{sec}$ and maximal rates greater than $30 \mathrm{spike} / \mathrm{sec}$. All units had exclusively simple action potentials, with no complex action potentials.

All units fired at their lowest rates during motionlessness and the same consummatory behaviors which would give irregular hippocampal slow waves (Figs. 6, 9; Table 1). As with motionlessness and consummatory behaviors, individual units varied little in rate across voluntary behaviors (Table 2), but there was considerable variation across units, with a range 
TABIE 1

Rates of Firint; on Various Consummatory Behavioks

\begin{tabular}{|c|c|c|c|c|c|}
\hline Cell & Drinking & Eating & Motionless & Grooming & Teeth chatter \\
\hline $110 \mathrm{~A}$ & 72 & 74 & & & \\
\hline $110 \mathrm{~B}$ & 68 & 77 & & 72 & \\
\hline $110 \mathrm{C}$ & 48 & 52 & 51 & & \\
\hline $110 \mathrm{D}$ & 12 & 10 & & 12 & \\
\hline $110 \mathrm{E}$ & 31 & & & 37 & \\
\hline $110 \mathrm{~F}$ & 55 & 46 & & 52 & 51 \\
\hline $110 \mathrm{G}$ & 25 & 22 & & 28 & 26 \\
\hline $110 \mathrm{H}$ & 28 & 38 & & & 21 \\
\hline $111 \mathrm{~A}$ & 41 & & & 36 & \\
\hline $121 \mathrm{~A}$ & 34 & & 16 & 40 & \\
\hline $122 \mathrm{~B}$ & 5 & & 13 & 8 & \\
\hline $122 \mathrm{C}$ & 17 & & & & \\
\hline $122 \mathrm{D}$ & 10 & 17 & & & \\
\hline $122 \mathrm{E}$ & 15 & 16 & 8 & 24 & \\
\hline $122 \mathrm{~F}$ & 11 & 27 & 7 & & \\
\hline $123 \mathrm{~A}$ & 35 & & 34 & 34 & \\
\hline
\end{tabular}

a Rates of firing are expressed in spikes per second.

TABLE 2

Rates of Firing on Various Voluntaky Behaviors ${ }^{a}$

\begin{tabular}{|c|c|c|c|c|c|c|}
\hline Cell & Walking & $\begin{array}{l}\text { Pick up } \\
\text { in hand, } \\
\text { struggle }\end{array}$ & $\begin{array}{l}\text { Jump out } \\
\text { of box }\end{array}$ & $\begin{array}{l}\text { Approach } \\
\text { food }\end{array}$ & $\begin{array}{l}\text { Follow } \\
E \text { 's hand }\end{array}$ & $\begin{array}{l}\text { Explore } \\
\text { novel } \\
\text { object or } \\
\text { inside of } \\
\text { running box }\end{array}$ \\
\hline $110 \mathrm{~A}$ & 88 & 95 & 71 & & & \\
\hline $110 \mathrm{~B}$ & 93 & 88 & & 93 & & 82 \\
\hline $110 \mathrm{C}$ & 73 & 85 & & & 80 & \\
\hline $110 \mathrm{D}$ & 28 & & 28 & 19 & 30 & \\
\hline $110 \mathrm{E}$ & 55 & & 48 & & 58 & \\
\hline $110 \mathrm{~F}$ & 96 & 96 & 96 & & & 96 \\
\hline $110 \mathrm{G}$ & 50 & & 56 & & 52 & 45 \\
\hline $110 \mathrm{H}$ & 56 & & 69 & 51 & 58 & 59 \\
\hline $111 \mathrm{~A}$ & 61 & & & & 57 & 62 \\
\hline $121 \mathrm{~A}$ & 59 & & 68 & 61 & 55 & \\
\hline $122 \mathrm{~B}$ & 26 & & & 22 & & 23 \\
\hline $122 \mathrm{C}$ & 37 & & & & & \\
\hline $122 \mathrm{D}$ & 34 & 41 & & & 24 & 40 \\
\hline $122 \mathrm{E}$ & 39 & 51 & & 40 & 35 & \\
\hline $122 \mathrm{~F}$ & 51 & 43 & 51 & 43 & & \\
\hline $123 \mathrm{~A}$ & 64 & & 68 & 66 & & 62 \\
\hline
\end{tabular}

* Rates of firing are expressed in spikes per second. 
WAI Y. I Fi

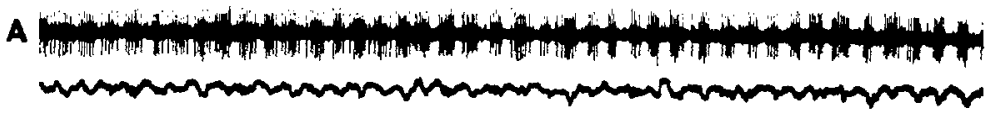

TEETH CHATIERINC

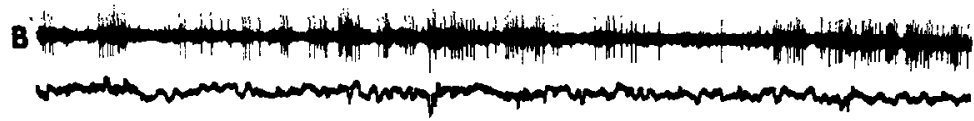

EXPLDPING CORHE. OF BOX

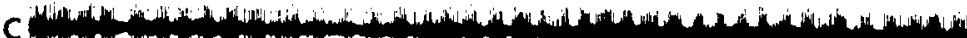

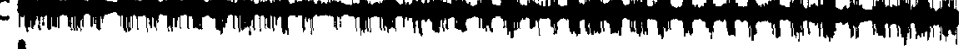

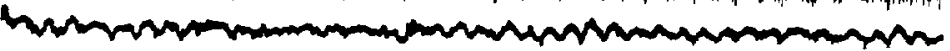

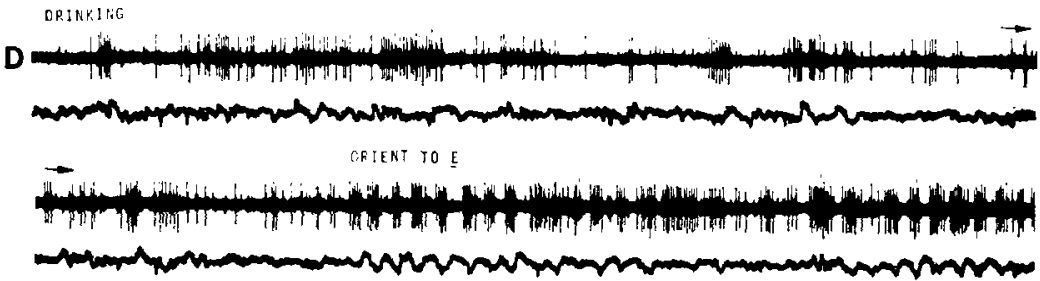

Frg. 6. Unit $110 \mathrm{G}$ and simultaneous slow waves from stratum moleculare of fascia dentata. This same unit is shown in Fig. 7 and 8. Note the fast, rhythmical firing of the unit with groups of action potentials in fixed phase xelation to the theta slow waves during walking, exploring, and orienting. Firing is slower and irregular during the consummatory behaviors of teeth chattering and drinking. The two lines of $D$ are continuous (marked by arrows in this and subsequent figures). Voltage calibration: $400 \mu \mathrm{v}$ for unit; $2 \mathrm{mv}$, for slow waves. Time: $1 \mathrm{sec}$.

of $24-96 \mathrm{spike} / \mathrm{sec}$ and a mean of 56.8 spike/sec (Table 4). In addition, there was a striking rhythmicity of firing during voluntary behaviors. This rhythmicity was due to the cells fring in groups of three to ten spikes, the groups being phase-locked to the theta rhythm in the slow waves. The phase relation of the group to the slow waves during theta remained constant for any one unit, but was variable across units. The relative amount of rhythmicity apparent upon visual inspection of firing records varied across units, some units displaying very obvious rhythmicity, other units showing more subtle rhythmicity. Three units showed no apparent rhythmicity.

Due to frequent problems with chewing and lapping artifact, acceptable data during bar pressing on continunus reinforcement was obtained in only seven cells. These data are summarized in Table 3 . The general finding was an overall low, irregular rate of firing (in the consummatory range), which persisted throughout the bar-pressing session from early to late reinforce- 
acquisition of bar pressing according to this view, because more orienting to the bar is needed during acquisition of the task.

O'Keefe and Dostrovsky (19) and O'Keefe and Nadel (personal communication) have proposed that theta is an accompaniment of an animal changing its position within its own cognitive map. This theory is also supported by the present results. One can assume that as long as the rat is at the bar pressing, it is not changing its locus in its cognitive map (it is always "at the bar"), while an approach to the reward delivery would be a change to "at the reward."

Routtenberg's (26) theory that hippocampal theta is a concomitant of an information processing state and irregular hippocampal activity is a concomitant of a response execution state is consistent with the present results, in that bar pressing is the exectition of a well-learned response. The theta which occurred during approach to the reward could be explained as a transitory stimulus processing state induced by the noise of the food magazine. Theta during bar pressing early in the acquisition of the response would be due to the information processing which accompanies learning. When we use a similar line of reasoning, these results are also consistent with Adey's view that theta is a concomitant of learning (1).

The results of the present study refute the hypothesis of Gray (13) that hippocampal theta of $7.7 \mathrm{~Hz}$ represents the registration of frustrative nonreward. According to Gray's hypothesis, switching from continuous reinforcement to fixed-ratio 50 and bar pressing under extinction should both elicit hippocampal $7.7 \mathrm{~Hz}$ theta. In neither of these two situations was theta found.

The problem with all of the theories mentioned here (including to some extent Vanderwolf's) is that they all imply internal mental states which at present cannot be tested directly. As a result, data can be made to fit many of the theories. Thus, no real choice can be made as to which of the theories discussed is most consistent with the present data. Until the appropriate internal mental states can be tested, Vanderwolf's theory has the advantage, at least in the rat, of being closest to a simple description of the behavior. The fact that in one instance in the present study slow-wave theta was recorded during strict consummatory behaviors and bar pressing could, among other things, suggest that there may be no general behaviorally definable correlate of theta. Putting theories aside, the present study has at least made the general neural and behavioral description of the hippocampal theta mode more clear. The significance of this mode of firing of theta cells is in the interaction of theta cells with other cells as this contributes to the function of the whole system. 


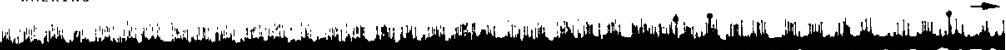

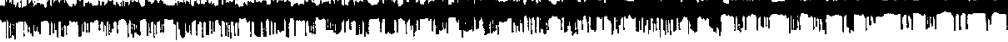
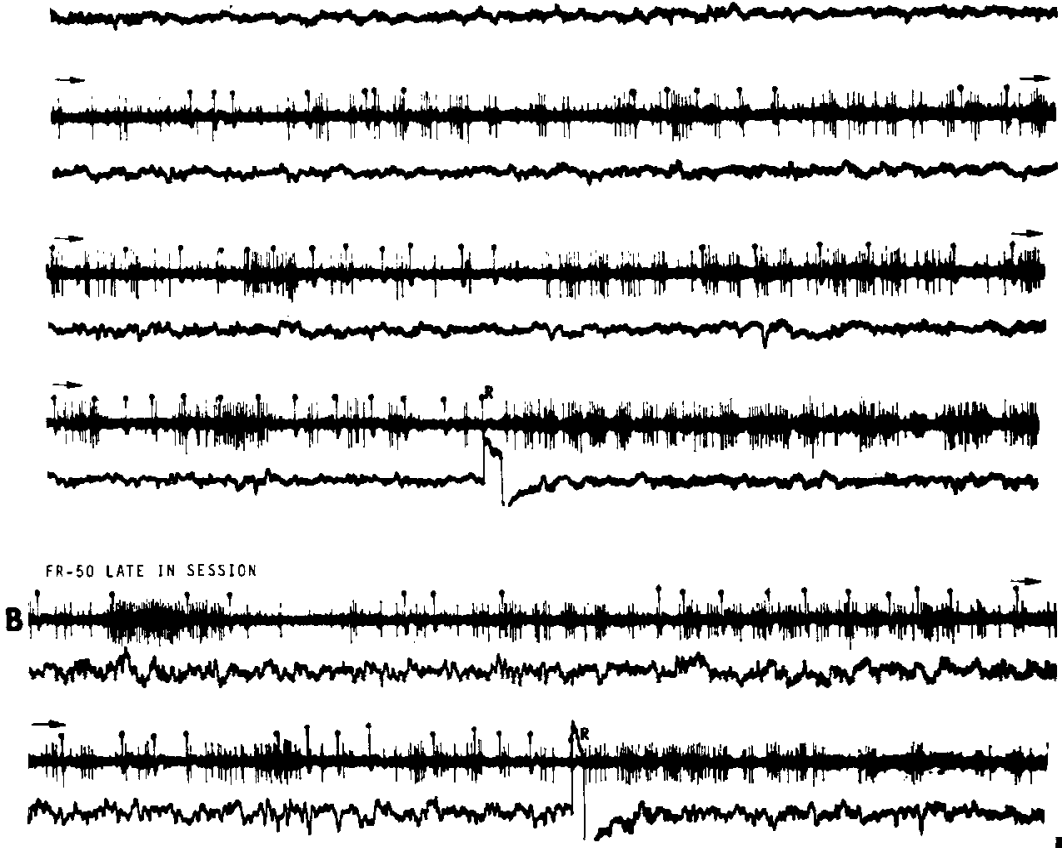

FIG. 8. Unit $110 \mathrm{G}$ and simultaneous slow waves during FR-50. Spikes topped with dots indicate bar presses, not action potentials. In A, animal is walking around and then starts bar pressing, this run coming early in the session. Bar pressing gives slow irregular unit firing and irregular slow waves. Note the brief increases in rate and rhythmicity of the unit after the reinforcement marker, while the animal was moving to the food chute to obtain reward, then a decline in unit rate as the animal begins consuming the reward. No fundamental differences were apparent from bar pressing early in the session and late in the session. Voltage calibration: $400 \mu \mathrm{v}$ for unit; $2 \mathrm{mv}$ for slow waves in $\mathrm{A} ; 1 \mathrm{mv}$ for slow waves in B. Time: $1 \mathrm{sec}$.

ments. The low, irregular firing rate was probably due in part to the fact that on continuous reinforcement the anmmals spent most of their time consuming the reward. Close analysis of firing records revealed that an increase in rate with theta rhythmicity, lasting between $0.4 \mathrm{sec}$ and $1 \mathrm{sec}$, occurred in units $110 \mathrm{C}, 110 \mathrm{E}, 110 \mathrm{G}$, and $123 \mathrm{~A}$ when the animal moved to the food chute to obtain reward (Fig. 7 ), and in unit $111 \mathrm{~A}$ when the animal approached the dipper and when he approached the bar, after drinking, for another press (Fig. 91. These brief increases were observed at no other times, including the time during which the rat was pressing.

Good data were obtained from all units on fixed-ratio 50 bar pressing 
$110 \mathrm{D}$
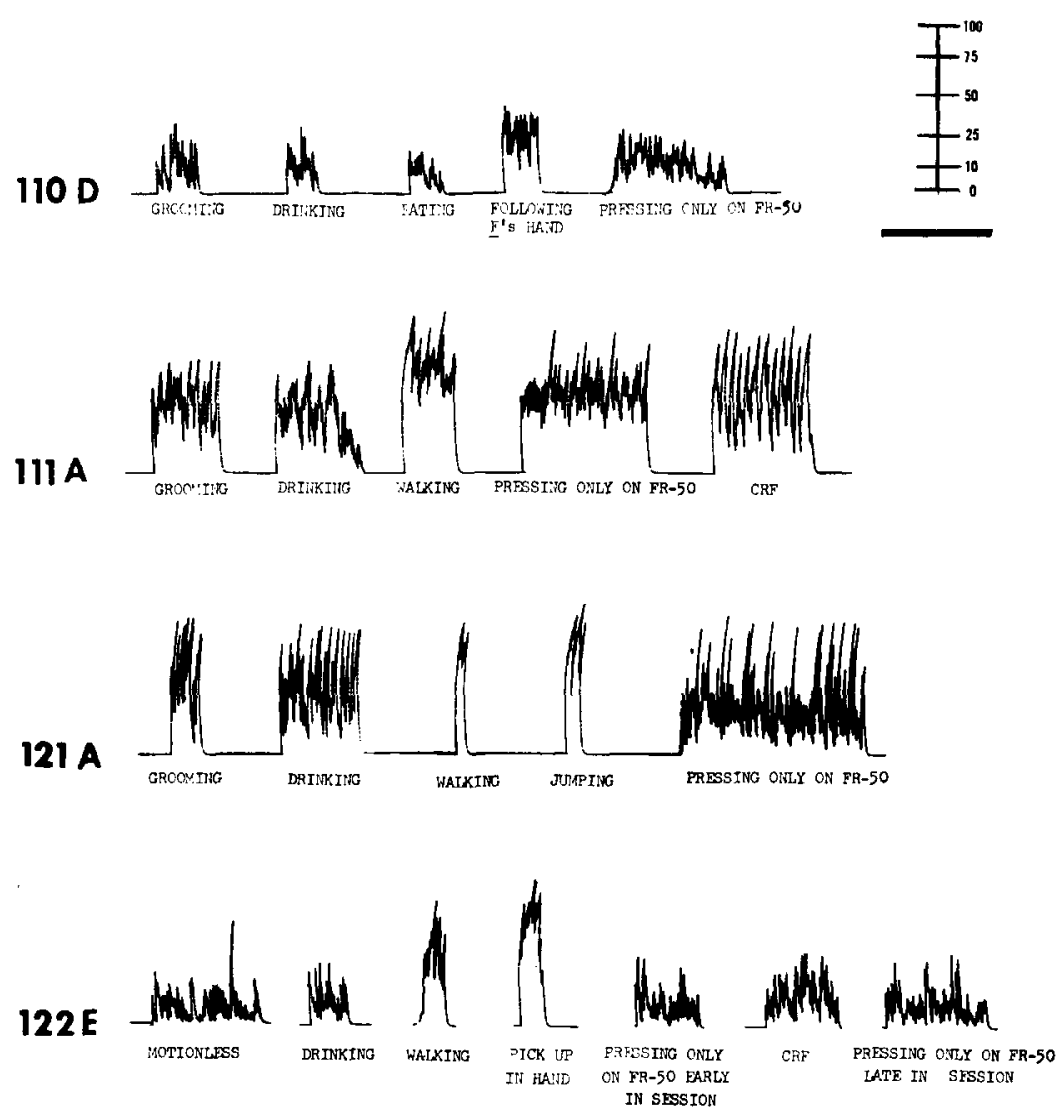

FIG. 9. Spike integration records for four different units. The decay of the integration had a time constant of $250 \mathrm{msec}$. These records show how low rates of firing on consummatory behaviors, CRF, and the pressing phase of FR-50 are sustained over long periods of the same behavior. High rates of firing on voluntary behaviors are similarly sustained over long periods of time. The FR-50 records were obtained by splicing together bar pressing only from five or more consecutive reinforcements of FR-50. The periudic increases in rate on CRF for unit $111 \Lambda$ wcrc corrclatcd with the animal approaching the dipper to obtain reward and approaching the bar for another press. Integrator calibration is in spikes (action potentials) per second. Time bar: $1 \mathrm{~min}$.

(Figs. 8, 9). All units showed irregular, low rates during the rat's pressing and during his consumption of the reward. Unit firing remained low and irregular throughout the bar-press session, from early to late reinforcements. For the pressing phase alone of fixed-ratio 50 , the range of rates was 8-80 spike/sec, with a mean of 33.1 spike/sec (Table 4 ). There were infrequent increases in rate and appearance of rhythmicity during pressing which were associated with postural adjustments. Eleven of the units (Table 4) showed 
TABLE +

Average Rates of Firini on Consummary Behaviors, Voluntary Behaviors, and the Pressing Phase of Fixed-Ratio 50

\begin{tabular}{|c|c|c|c|c|c|}
\hline Cell & Location & $\begin{array}{l}\text { Consum- } \\
\text { matory } \\
\text { behaviors }\end{array}$ & $\begin{array}{l}\text { Voluntary } \\
\text { behaviors }\end{array}$ & $\begin{array}{l}\text { Fixed- } \\
\text { ratio } \\
50 \text { press }\end{array}$ & $\begin{array}{c}\text { Increase in } \\
\text { firing rate } \\
\text { and rhyth- } \\
\text { micity with } \\
\text { approach to } \\
\text { reward on } \\
\text { fixed-ratio } \\
50 ?\end{array}$ \\
\hline $110 \mathrm{~A}$ & $\mathrm{CA} 4$ & 7.3 & 85 & 80 & No \\
\hline $110 \mathrm{~B}$ & CA4 & 72 & 89 & 65 & les \\
\hline $110 \mathrm{C}$ & CA1 & 49 & 79 & 61 & Ves \\
\hline $110 \mathrm{D}$ & Fascia dentata & 11 & 26 & 16 & $\mathrm{No}$ \\
\hline $110 \mathrm{E}$ & CA 3 & 34 & 54 & 41 & Yes \\
\hline $110 \mathrm{~F}$ & Fascia dentata & 51 & 96 & 36 & Yes \\
\hline $110 \mathrm{G}$ & Fascia dentata & 25 & 51 & 24 & Yes \\
\hline $110 \mathrm{H}$ & Fascia dentata & 29 & 59 & 32 & Yes \\
\hline $111 \mathrm{~A}$ & $\mathrm{CA} 3$ & 38 & 60 & 45 & Yes \\
\hline $121 \mathrm{~A}$ & $\mathrm{CA} 1$ & 30 & 61 & 25 & No \\
\hline $122 \mathrm{~B}$ & $\mathrm{CA} 1$ & 9 & 24 & 12 & Yes \\
\hline $122 \mathrm{C}$ & $\mathrm{CA} 1$ & 17 & 37 & 10 & No \\
\hline $122 \mathrm{D}$ & CA3 & 1.3 & 35 & 8 & No \\
\hline $122 \mathrm{E}$ & CA.3 & 16 & 41 & 11 & Yes \\
\hline $122 \mathrm{~F}$ & Fascia dentata & 15 & 47 & 18 & Yes \\
\hline $123 \mathrm{~A}$ & CA1 & 34 & 65 & 46 & les \\
\hline \multicolumn{2}{|c|}{ Mean for all cells } & 32.2 .5 & 56.81 & 33.125 & \\
\hline
\end{tabular}

${ }^{a}$ Rates of firing are expressed in spikes per second.

increases in rate and appearance of rhythmicity for 0.4-2 sce during the rat's approach to the food chute or the water dipper to obtain reward (Fig. 8).

Table 4 presents the average rates of all units in this study for consummatory behaviors, voluntary behaviors, and the pressing phase of fixed-ratio 50 . As can be seen there, the rates for pressing on fixed-ratio 50 for individual cells were much closer in magnitude to the rates on consumnatory behaviors than on voluntary behaviors. These data are presented graphically in Fig. 10. The relative rate (fast or slow) for any particular unit, in relation to all units, was the same in all three categories of behavior. Thus, a "fast" unit was always fast; a "slow" unit, always slow (in relation to other units). There was no obvious relation between this fixed relative speed of a unit and its anatomical location. The difference over all units between mean rates on consummatory behaviors and mean rates on the pressing plase of fixed-ratio 


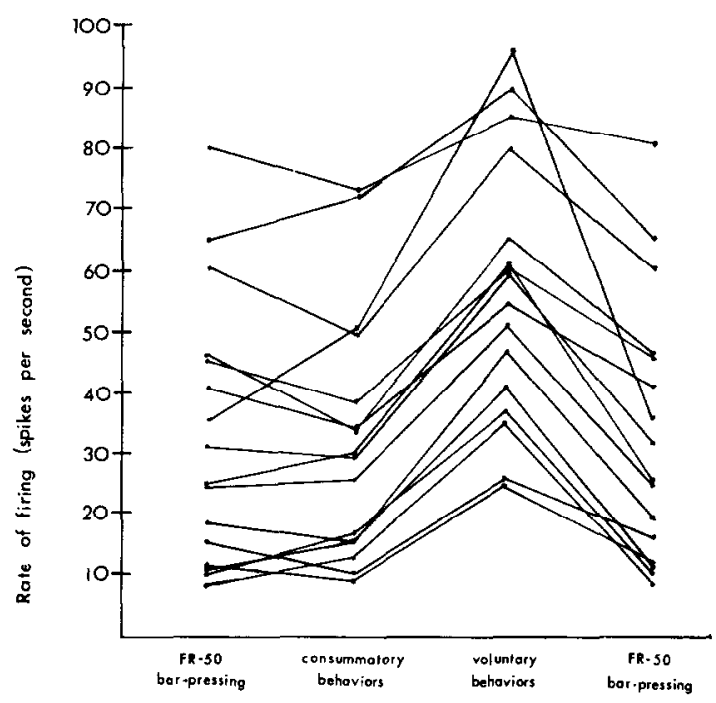

FIg. 10. Summary of data in Table 4 for average rates of firing on voluntary behaviors, consummatory behaviors, and the pressing phase of FR-50. Each line, with four data points, represents one unit. FR-50 is duplicated for ease of comparison with both consummatory behaviors and voluntary behaviors.

50 was not significant $(t(15)=0.46,0.60<P<0.70)$, while mean rates on voluntary behaviors were significantly different from consummatory behavior rates $(t(15)=11.63, P<0.001)$, and bar-pressing phase tates of fixed ratio $50(t(15)=7.34, P<0.001)$.

\section{DISCUSSION}

The findings in this study of the general behavioral correlates of the firing of theta cell hippocampal neurons supplement those of a previous paper (24) in showing that a particular hippocampal neuron type will fire reliably in a particular fashion during particular overt behaviors. Theta cells have only two basic firing modes, an automatic mode and a theta mode. For a given cell the rate of firing in each mode was the same regardless of exactly what behavior was being performed by the rat.

The amount of recordable slow wave theta was found to vary considerably with the monopolar electrode placement within the hippocampus. However, theta cells from all regions of the dorsal hippocampus responded alike. Therefore, some part of the theta mechanism is uniform throughout the dursal hippocampus.

The rates of firing of these neurons present a means of describing the theta mode in a quantified way, susceptible to statistical analysis. This could be of great advantage in untangling the disputes which have resulted concerning the behavioral correlates of theta based solely on subjective 
visual inspection of slow-wave records. While the amount of rhythmicity of firing of theta neurons was not rigorously analyzed in this study, it is conceivable that this, too, could prove a fruitful method of quantifying results.

Another advantage of studying neuronal theta responses which this study revealed was the discovery of reliable, brief changes in firing rate (less than $1 \mathrm{sec}$ long). such as occurred during approach to the reinforcement during bar pressing, which are so quick that the presence of the response would have been at best ambiguous had only slow wave data been used.

The slow-wave theta rhythm in Ammon's horn is no cloubt due to postsynaptic potentials in pyramidal cells (11) ${ }^{3}$ The relation of the theta cells to the generation of these slow waves is not established and possible relations will not be argued here. However, whatever the mechanism, these theta cells are clearly an important part of it, if for no other reason than they are like the theta cells of septum. Theta cclls are present in the rat in some locations where there is no theta rhythm (septum) or little theta rhythm (fascia dentata) (25). A regular slow wave theta rhythm does not exist in prinate hippocampus. This raises the possibility that theta cells exist in primates, with the same mechanisms as in other mammals. Indeed, even in rats we define theta mode by rate, rather than pattern.

All of the theta cells in this study exhibited the same behavioral correlate, with the same change in firing pattern. It seems uneconomical for so many neurons to signal the same thing. There may well be information coded in theta cells in ways which this study dicl not appreciate. A kind of lateral inhibition has been suggested (2t). Evidence for more information being conveyed in the theta mode than has been appreciated in this study include Vanderwolf's (32) findings that amplitude and frequency (in cycles per second) of theta rhythm may change independently and Adey, Dunlop, and Hendrix (1) findings that the phase relation of theta rhythm in hippocampus and entorhinal cortex differ in different behaviors. Vanderwolf (31) and Elazar and Adey (9) have also shown that different frequencies of the theta rlythm have different behavioral correlates. In the present study, a two-state mechanism was apparent. Hippocampal slow waves and theta units either exhibited 8-10 cycle/sec theta or irregular activity, with $+7 \mathrm{cycle} / \mathrm{sec}$ theta and intermediate rates of firing only being observed occasionally as a short-lived transition state. This may be a peculiarity of the rat, as other animals can maintain a low frequency theta rhythm for many seconds ( 1 ).

Bar-Pressing. The unit and slow-wave responses during bar pressing

${ }^{3}$ In fascia dentata, subiculum, presubiculum, parasubiculum, and entorhinal cortex where a slow-wave theta rhythm can also be recorded, we know of no data bearing on its mechanism and can only argue that it is due to postsynaptic potentials because most slow waves are. 
clearly indicate that this response, when well learned, is not accompanied by theta. No basic differences were found between bar pressing for food reward or bar pressing for water reward, or between bar pressing early or late in a session, or for bar pessing on extinction. Motivational mode and level thus do not seem to be involved in any obvious way here with the theta mode.

The irregular, non-theta mode during actual bar pressing agrees with the results of Pond and Schwartzbaum (23) and Lopes da Silva and Kamp (18). The results of the present study differ from those of Dalton and Black (8) and Wishaw and Vanderwolf (33). Both of the latter studies used very few training trials, whereas the former two studies used longer training periods (although not as long as the present study). From the descriptions of Lopes da Silva and Kamp (18) and Grastyan et al. (12), of the gradual loss of theta over training, it seems likely that the results of Dalton and Black, and Venderwolf and Wishaw, were due to too short a training period.

Vanderwolf's suggestion for the failure to find theta on the basis of electrode placement (31) does not hold in this study. Unit responses were the same in all regions of the hippocampus, and slow-wave placements which did yield good theta during all voluntary behaviors showed clearly irregular slow waves during the "intense" movement on fixed-ratio 50 bar pressing. There is, however, the exceptional slow-wave result with animal 110 in which theta was observed in CA4 during bar pressing. But since this locus also gave theta during some consummatory behaviors, and on all other recording days in different hippocampal loci in this animal irregular slow waves and unit responses were seen during bar pressing, this lone finding does not clearly support Vanderwolf. The finding in animal 122 of one electrode site which gave slow-wave theta during the beginning of fixedratio 50 pressing is also difficult to interpret, as the same animal again gave irregular slow waves during bar pressing from all other electrode sites, and showed no similar evidence of theta in any of its five units.

Implication for Theories of the Gencral Behavioral Correlale of Hippocampal Theta. It is apparent, then, from all unit data and the majority of slow-wave data from this study, that a clearly voluntary, conditionable behavior can be associated with an irregular, desynchronized hippocampal theta mechanism. While hippocampal theta does appear to be a concomitant of voluntary movement in natural, unlearned behaviors, Vanderwolf's theory must be revised to allow for some learned automatic behaviors. Well-learned bar pressing does indeed show certain similarities to other nonlearned automatic behaviors like eating, drinking, and grooming in that these latter behaviors and well-learned bar pressing are all repetitive, fixerl patterns of behavior.

The approach to the food and water delivery to obtain reward was ac- 
companied by a theta mode in most units. Presumably this approach was as well learned and utilized and as much of a fixed pattern as the bar pressing.

Similarly, a rat's approaching a goal box (1), jumping to aroid shock (31), and forced running in a running wheel (33), are all theta mode behaviors. Thus some well-learncd behaviors arc in the theta mode and others are not. The basis for this striking difference is not clear. It is surprising that no intermediate states have been observed.

Proponents of the voluntary movement hypothesis might raise the point that the reason no theta was found during bar pressing in this study is that the magnitude of movement by the animal is not large enough to elicit theta once the response becomes very well learned. While this might be valid for continuous reinforcement, fixed-ratio 50 bar-pressing was, as mentioned before, a very rapid, intense shaking of the whole upper torso of the animal. This movement on fixed-ratio 50 is at least as great in terms of displacement and effort as that of manipulating a food pellet during eating, a behavior which Vanderwolf (31) reported and with which we agree. is always accompanied by theta.

The results of this study should also be discussed in terms of other current theories of the behavioral correlate of hippocampal theta. Bennett (5) and Grastyan et al. (12) have explained theta as a concomitant of overt orienting to environmental stimuli. While this explanation of the theta mechanism is perhaps more obvious from studying cats than rats $(5,34)$, it can be made to fit the general findings with rats, since rats do exhibit theta during orienting head and body movements, and continuous orienting can be assumed to occur during walling, following or approaching an object, jumping, exploring, etc. Motionlessness and consummatory behaviors might not require orienting since these behaviors can be inagined to be controlled by internal programs, not requiring continued guidance by external stimuli. The present finding of no theta during bar pressing is consistent with this formulation if it is assumed that the bar-press response was so well learned that no orienting was needed to the meaningful stimulus (the bar) once the rat had its paw on it. The bar pressing was automatic once the meaningful stimulus was obtained (as in consummatory behaviors). Going over and getting the reward would, however, require orientation to the reward, and thus theta was seen in this situation. The return to the bar after consuming the reward did not give a similar brief theta response except in the animal on continuous reinforcement water reward, because continuous reinforcement on water was the only situation in which a quick, direct approach to the bar was made. "Theta would be seen during the early stages of

4 On continuous reinforcement for fool, the animal had its paw on the bar continuously, and on fixed-ratio 50 for food or water, the animal slowly "drifted" back to the bar. 
acquisition of bar pressing according to this view, because more orienting to the bar is needed during acquisition of the task.

O'Keefe and Dostrovsky (19) and O'Keefe and Nadel (personal communication) have proposed that theta is an accompaniment of an animal changing its position within its own cognitive map. This theory is also supported by the present results. One can assume that as long as the rat is at the bar pressing, it is not changing its locus in its cognitive map (it is always "at the bar"), while an approach to the reward delivery would be a change to "at the reward."

Routtenberg's (26) theory that hippocampal theta is a concomitant of an information processing state and irregular hippocampal activity is a concomitant of a response execution state is consistent with the present results, in that bar pressing is the exectition of a well-learned response. The theta which occurred during approach to the reward could be explained as a transitory stimulus processing state induced by the noise of the food magazine. Theta during bar pressing early in the acquisition of the response would be due to the information processing which accompanies learning. When we use a similar line of reasoning, these results are also consistent with Adey's view that theta is a concomitant of learning (1).

The results of the present study refute the hypothesis of Gray (13) that hippocampal theta of $7.7 \mathrm{~Hz}$ represents the registration of frustrative nonreward. According to Gray's hypothesis, switching from continuous reinforcement to fixed-ratio 50 and bar pressing under extinction should both elicit hippocampal $7.7 \mathrm{~Hz}$ theta. In neither of these two situations was theta found.

The problem with all of the theories mentioned here (including to some extent Vanderwolf's) is that they all imply internal mental states which at present cannot be tested directly. As a result, data can be made to fit many of the theories. Thus, no real choice can be made as to which of the theories discussed is most consistent with the present data. Until the appropriate internal mental states can be tested, Vanderwolf's theory has the advantage, at least in the rat, of being closest to a simple description of the behavior. The fact that in one instance in the present study slow-wave theta was recorded during strict consummatory behaviors and bar pressing could, among other things, suggest that there may be no general behaviorally definable correlate of theta. Putting theories aside, the present study has at least made the general neural and behavioral description of the hippocampal theta mode more clear. The significance of this mode of firing of theta cells is in the interaction of theta cells with other cells as this contributes to the function of the whole system. 


\section{SUMMARY}

Hippocampal slow waves and firing of theta cells were investigated during voluntary and automatic behaviors of the rat, including bar pressing on continuous reinforcement and fixed-ratio 50 . Voluntary behaviors (walking. orienting, postural adjustments, approaching food or water, following the experimenter's hand, jumping, and exploring) were accompanied by theta in the slow waves and fast, rhythmical firing in the theta cells. For a given cell the rates of firing were similar for all voluntary behaviors. Automatic behaviors (eating, drinking, teeth chattering, grooming, vomiting, and yawning) were accompanied by irregular slow-wave activity and slow, irregular firing in the theta celis. For a given cell the rates of firing were similar for all automatic behaviors. Electrode placement within the hippocampus was critical with regard to how much slow-wave theta could be recorded during voluntary behaviors, whereas theta cells throughout the hippocampus were identical in the form of their firing. Bar pressing on both continuous reinforcement and fixed-ratio 50 gave clearly non-theta responses in both units and slow waves. Somc well-learned voluntary behaviors can become automatic and are not in the theta mode.

\section{REFERENCES}

1. Adey, W. R., C. W. Duriop, and C. E. Hendrix. 1960. Hippocampal slow waves: Distribution and phase relationships in the course of approach learning. Arch. Neurol. 3 : 74-90.

2. Adey, W. R., and D. O. Walter. 1963. Application of phase detection and averaging techniques in computer analysis of EEG records in the cat. Exp. Neurol. 7: 282-293.

3. Adey, W. R., D. O. Walter, and C. E. Hendrix, 1961. Computer techniques in correlation and spectral analysis of cerebral slow waves during discriminative behavior. Exp. Neurol. 3 : 501524.

4. Adey, W. R., D. O. Walter, and D. F. Lindsley. 1962. Subthalamic lesions: Effects on learned behavior and correlated hippocampal and subcortical slowwave activity. Arch. Neurol. 6: 194-207.

5. BennetT, T. L. 1971. Hippocampal theta activity and behavior-A review. Commun. Bchav. Biol. Part A, 6:37-48.

6. Black, A. H., G. A. Young, and C. Batenchuk. 1970. Avoidance training of hippocampal theta waves in flaxadilized dogs and its relation to skeletal movement. I. Comp. Physiol. Psychol. $70: 15-24$.

7. Dalton, A. J. 1969. Discriminative conditioning of hippocampal electrical activity in curarized dogs. Commun. Brhaz'. Biol. 3: 283-287.

8. Dalton, A, and A. H. Black. 1968. Hippocampal electrical activity during the operant conditioning of movement and refraining from movement. Commun. Bchav. Biol. 2: 267-273.

9. ElAZAR, Z., and W. R. AdEY. 1967. Spectral analysis of low frequency components in the electrical activity of the hippocampus during learning. Electrocncophalogr. Clin. Nourophysiol. 23 : 225-240. 
10. Ford, J. G., F. J. Bremner, and W. R. Richie. 1970. The effect of hours of food deprivation on hippocampal theta rhythm. Neuropsychologia 8: 65-73.

11. FuJita, Y., and T. Sato. 1964. Intracellular records from hippocampal pyramidal cells in rabbit during theta rhythm activity. $J$. Neurophysiol. $27: 1011-1025$.

12. Grastyan, E., K. Lissak, I. Madrasy, and H. Donhoffer. 1959. Hippocampal electrical activity during the development of conditioned reflexes. Electroencephalogr. Clin. Neurophysiol. $11: 409-480$.

13. Gray, J. A. 1970. Sodium amobarbital, the hippocampal theta rhythm, and the partial reinforcement extinction effect. Psychol. Rev. 77: 465-480.

14. Irmis, F., J. Madlafousek, and Z. Hlinak. 1970. Hippocampal electrical activity in course of sexual behavior of male rats. Physiologia Bohemoslov 19: 83-87.

15. Irmis, F., T. Radil-Weiss, J. Lat, and I. Krekule. 1970. Interindividual differences in hippocampal theta activity during habituation. Electrocncephalogr. Clin. Neurophysiol. 28 : 24-31.

16. Iто, M. 1966. Hippocampal electrical correlates of self-stimulation in the rat. Electroencephalogr. Clin. Neurophysiol. $21: 261-268$.

17. Iто, M., and J. OL.Ds. 1971. Unit activity during self-stimulation behavior. $J$. Neurophysiol. 34 : 263-273.

18. Lopes DA Silva, F. H., and A. Kamp. 1969. Hippocampal theta frequency shifts and operant behavior. Electroencephalogr. Clin. Neurophysiol. 26: 133-143.

19. O'Kelfe, J., and J. Dustrovsky. 1971. The hippocampus as a spatial map. Preliminary evidence from unit activity in the freely moving rat. Brain Res, 34: $171-175$.

20. Paxinos, G., and D. Bindra. 1970. Rewarding intracranial stimulation, movement, and the hippocampal theta rhythm. Physiol. Behav. 5: 227-231.

21. Pickenhain, L., and F. Klingberg. 1967. Hippocampal slow wave activity as a correlate of basic behavioral mechanisms in the rat. Progr. Brain Res. 27: 218-227.

22. Pond, F., and J. S. Schwartzbaum. 1970. Hippocampal electrical activity evoked by rewarding and aversive brain stimulation in rats. Commun. Bchav. Biol. 5: 89-103.

23. Pond, F., and J. S. Schwartzbaum. 1972. Interrelationships of hippocampal EEG and visual evoked responses during appetitive behavior in rats. Brain Res. 43 : $119-137$.

24. Ranck, J. B., JR. 1973. Studies on single neurons in dorsal hippocampal formation and septum in unrestrained rats. Part I. Behavioral correlates and firing repertoire. Exp. Ncurol. 40:461-555.

25. Routtenderc, A. 1968. Hippocampal correlates of consummatory and observed behavior. Physiol. Behav. 3: 533-535.

26. Routtenberg, A. 1971. Stimulus processing and response execution: A neurobehavioral theory. Physiol. Behav. 6: 589-596.

27. Routtenberg, A., E. B. Zechmeister, and C. Benton. 1970. Hippocampal activity during memory disruption of passive avoidance by electroconvulsive shock. Lif $\mathcal{C}$ Sci. 9: 909-918.

28. SAINSBURY, R. S. 1970. Hippocampal activity during natural behavior in the guinea pig. Physiol. Behav. 5: 317-324.

29. Teitelbaum, H., and W. L. McFarland. 1971. Power spectral shifts in hippocampal EEG associaterl with conditioned locomotion in the rat. Physiol. Behar'. $7: 545-549$.

30. VANDERWOLF, C. H. 1969. Hippocampal electrical activity and voluntary movement in the rat. Elcctrocncephalogr. Clin. Nourophysiol. 26: 407-418. 
31. Vandikwolf, C. H. 1971. Limbic diencephalic mechanisms of voluntary movenent. Psychol. Rew. 78: 83-113.

32. Whisina, I. Q., and C. H. VANberwol.F. 1971. Hippocampal EEG and behavior: Effects of variation in body temperature and relation of EEG to vibrissae movement, swimming, and shivering. Physiol. Bchav. 6: 391-397.

33. WinsHAw, I. Q., and C. H. VANDERWoLf. 1973. Hippocampal EEG and behavior: Changes in amplitude and frequency of RSA (theta rhythm) associated with spontaneous and learned movement patterns in rats and cats. Behavioral Biol. 8: $461-484$.

34. Winsox, J. 1972. Inter-species differences in the occurrence of theta. Bchavioral Biol. 7 : 479-487.

35. Yoshin, N., M. Shimokochi, K. Мiуamoto, and M. Ito. 1966. Studies on the neural basis of behavior by continuous frequency analysis of EEG. Progr. Brain Res. 21A : $217-250$. 